\title{
L-fuzzy pre-proximities, L-fuzzy filters and $L$-fuzzy grills
}

\author{
A. A. Ramadan ${ }^{1}$, M. A. Usama ${ }^{1}$ and A. A. Abd El-Latif2,3*
}

*Correspondence:

abdultif@ub.edu.sa;

ahmeda73@yahoo.com

${ }^{3}$ High Institute of Computer King Marriott, P.O. Box 3135 ,

Alexandria, Egypt

Full list of author information

is available at the end of the

article

\begin{abstract}
This article gives results on fixed complete lattice L-fuzzy pre-proximities, L-fuzzy grills and $L$-fuzzy filters. Moreover, we investigate the relations among the $L$-fuzzy pre-proximities, L-fuzzy grills and L-fuzzy filters. We show that there is a Galois correspondence between the category of separated $L$-fuzzy grill spaces and that of separated $L$-fuzzy pre-proximity spaces. We introduced the local function associated with L-fuzzy grill and L-fuzzy topology and studied some of its properties. Finally, we build an L-fuzzy topology for the corresponding L-fuzzy grill by using local function.
\end{abstract}

Keywords: Complete lattice, Implicator, L-fuzzy pre-proximity, L-fuzzy grills, L-fuzzy filters, Galois correspondence, L-fuzzy topology

Mathematics Subject Classification: 03E72, 54A40, $18 \mathrm{~B} 30$

\section{Introduction}

Proximity is an important concept in topology and it can be considered either as axiomatizations of geometric notions, close to but quite independent of topology, or as convenient tools for an investigation of topological spaces. Hence proximity has close relations with topology, uniformity and metric. With the development of topology, the theory of proximity makes a massive progress. In the framework of $L$-topology, many authors generalized the crisp proximity to $L$-fuzzy setting. For example, in [1], Ghanim et al. introduced the concept of S-quasi-proximities on $[0,1]^{X}$ and in [2], Shi studied $\mathrm{S}$-quasi-proximities on $L^{X}$ and pointwise S-quasi-proximities. Katsaras [3-5] introduced quasi-proximity in [0,1]-fuzzy set theory. Subsequently, Liu [6], Artico and Moresco [7] extended it into $L$-fuzzy set theory. In recently Yue and Shi extended the proximity theory of $L$-topology to $L$-fuzzy topology, see [8]. As an extension of Katsaras's definition, Kim and Min[9] introduced $L$-fuzzy proximities on strictly two-sided, commutative quantales $L$ in view points of Höhle fuzzy topology [10,11]. Thron [12] carried out an extensive study of proximity structures with grills playing a central role.

In this paper, we introduce more properties of $L$-fuzzy pre-proximities , $L$-fuzzy grills and $L$-fuzzy filters. Moreover, we investigate the relations among the $L$-fuzzy pre-proximities , $L$-fuzzy grills and $L$-fuzzy filters. We show that there is a Galois correspondence between the category of separated $L$-fuzzy grill spaces and that of separated $L$-fuzzy pre-proximity spaces. We introduce the local function associated with $L$-fuzzy grill and

(c) The Author(s) 2020. Open Access This article is licensed under a Creative Commons Attribution 4.0 International License, which permits use, sharing, adaptation, distribution and reproduction in any medium or format, as long as you give appropriate credit to the original author(s) and the source, provide a link to the Creative Commons licence, and indicate if changes were made. The images or other third party material in this article are included in the article's Creative Commons licence, unless indicated otherwise in a credit line to the material. If material is not included in the article's Creative Commons licence and your intended use is not permitted by statutory regulation or exceeds the permitted use, you will need to obtain permission directly from the copyright holder. To view a copy of this licence, visit http:// creativecommons.org/licenses/by/4.0/. 
$L$-fuzzy topology and study some of its properties. Finally, we build an $L$-fuzzy topology for the corresponding $L$-fuzzy grill by using local function.

The content of the paper is organized as follows. In Sect. 2, we recall some fundamental concepts and related definitions of $L$-fuzzy closure operators, $L$-fuzzy interior operators, $L$-fuzzy pre-proximities, $L$-fuzzy grills and $L$-fuzzy filters . In Sect. 3, we investigates the relations among the $L$-fuzzy pre-proximities and $L$-fuzzy grills. In Sect. 4 , we investigates the relations among the $L$-fuzzy pre-proximities and $L$-fuzzy filters. In Sect. 5, there is a Galois correspondence between the category of $L$-fuzzy pre-proximity spaces and that of $L$-fuzzy grill spaces. In Sect. 6, we introduce the local function associated with $L$-fuzzy grill and $L$-fuzzy topology and study some of its properties. Also, we build an $L$-fuzzy topology for the corresponding $L$-fuzzy grill by using local function.

\section{Preliminaries}

Throughout the text we consider $(L, \leq, \vee, \wedge)$ (or $L$ in short) as fixed complete lattice, that is a lattice in which the suprema (joins) and infima (meets) for all subfamilies $K \subseteq L$ exist. In particular, the top $\top$ and the bottom $\perp$ elements in $L$ exist and $\top \neq \perp$. We use notation $\vee$ and $\wedge$ to denote, respectively, infima and suprema of finite families of the elements of the lattice having notation $\bigvee$ and $\bigwedge$ for the case when these families are arbitrary. We will additionally request the lattice $L$ to be completely distributive, that is satisfying the first infinite distributive law of finite meets over arbitrary joins:

$$
x \wedge \bigvee_{i \in \Gamma} y_{i}=\bigvee_{i \in \Gamma}\left(x \wedge y_{i}\right), \quad \forall x, y_{i} \in L
$$

If $a \leq b$ or $b \leq a$, for each $a, b \in L$, then $L$ is called a chain. A lattice $L$ is called an order dense chain if for each $a, b \in L$ such that $a<b$, there exists $c \in L$ such that $a<c<b$.

Definition 2.1 [13-16] An implicator on a lattice $L$ is a mapping $\rightarrow: L \times L \rightarrow L$ defined by $x \rightarrow y=\bigvee\{z \in L \mid x \wedge z \leq y\}$, such that:

(1) $\top \rightarrow x=x, x \rightarrow \top=\top$ and $\perp \rightarrow x=\top$,

(2) If $y \leq z$, then $x \rightarrow y \leq x \rightarrow z$ and $z \rightarrow x \leq y \rightarrow x$,

(3) $x \leq y$ iff $x \rightarrow y=\top$ and $x \wedge y \leq z$ iff $x \leq y \rightarrow z$ for $x, y, z \in L$,

(4) $x \rightarrow(y \wedge z)=(x \rightarrow y) \wedge(x \rightarrow z)$ and $(x \vee y) \rightarrow z=(x \rightarrow z) \wedge(y \rightarrow z)$,

(5) $(x \wedge y) \rightarrow z=x \rightarrow(y \rightarrow z)=y \rightarrow(x \rightarrow z)$,

(6) $x \wedge(x \rightarrow y) \leq y$ and $y \leq x \rightarrow(x \wedge y)$ and $(x \rightarrow y) \rightarrow y \geq x$,

(7) $(x \rightarrow \perp) \rightarrow(y \rightarrow \perp)=y \rightarrow x$,

(8) $x \wedge y=(x \rightarrow(y \rightarrow \perp)) \rightarrow \perp$, and $x \vee y=(x \rightarrow \perp) \rightarrow y$.

From (7) and (1) we have the following important double negation property:

$$
(x \rightarrow \perp) \rightarrow \perp=x .
$$

Thus $x \rightarrow \perp$ is an order-reversing involution on $L$ and in the following we write $x^{*}=x \rightarrow \perp$. Referring to the properties of the implicator we see that De Morgan laws 
hold in the lattice with involution $\left(L, \leq, \vee, \wedge,{ }^{*}\right)$ determined by an implicator. In what follows $(L, \leq, \vee, \wedge, \rightarrow)$ is a complete lattice endowed with an implicator.

For $\alpha \in L, f \in L^{X}$, we denote $(\alpha \rightarrow f),(\alpha \wedge f)$ and $\quad \alpha_{X} \in L^{X} \quad$ as $(\alpha \rightarrow f)(x)=\alpha \rightarrow f(x),(\alpha \wedge f)(x)=\alpha \wedge f(x)$, and $\alpha_{X}(x)=\alpha$,

$$
\top_{x}(y)=\left\{\begin{array}{ll}
\top, & \text { if } y=x, \\
\perp, & \text { otherwise },
\end{array} \top_{x}^{*}(y)= \begin{cases}\perp, & \text { if } y=x \\
\top, & \text { otherwise }\end{cases}\right.
$$

A fuzzy point $x_{t}$ for $t \in L_{\perp}=L-\{\perp\}$ is an element of $L^{X}$ such that, for $y \in X$ :

$$
x_{t}(y)=\left\{\begin{array}{lll}
t, & \text { if } & x=y \\
\perp, & \text { if } & x \neq y
\end{array}\right.
$$

The set of all fuzzy points in $X$ is denoted by $\operatorname{Pt}(X)$.

Definition 2.2 [12]A map $\mathcal{G}: L^{X} \rightarrow L$ is called an $L$-fuzzy grill on $X$ if $\mathcal{G}$ satisfies the following conditions for all $f, g \in L^{X}$ :

$$
\begin{array}{ll}
\text { LG1 } & \mathcal{G}\left(\perp_{X}\right)=\perp, \mathcal{G}\left(\top_{X}\right)=\top, \\
\text { LG2 } & \text { If } f \leq g, \text { then } \mathcal{G}(f) \leq \mathcal{G}(g), \\
\text { LG3 } & \mathcal{G}(f \vee g) \leq \mathcal{G}(f) \vee \mathcal{G}(g) .
\end{array}
$$

The pair $(X, \mathcal{G})$ is called an $L$-fuzzy grill space. An $L$-fuzzy grill space is called:

(1) Stratified if $\mathcal{G}(\alpha \rightarrow f) \leq \alpha \rightarrow \mathcal{G}(f)$ for all $f \in L^{X}$ and $\alpha \in L$.

(2) Separated if $\mathcal{G}\left(\top_{x}^{*}\right)=\perp$, for all $x \in X$.

(3) Alexandroff if $\mathcal{G}\left(\bigvee_{i \in \Gamma} f_{i}\right)=\bigvee_{i \in \Gamma} \mathcal{G}\left(f_{i}\right), \forall\left\{f_{i}: i \in \Gamma\right\} \subseteq L^{X}$.

Let $\left(X, \mathcal{G}_{X}\right)$ and $\left(Y, \mathcal{G}_{Y}\right)$ be $L$-fuzzy grill spaces. $\varphi:\left(X, \mathcal{G}_{X}\right) \rightarrow\left(Y, \mathcal{G}_{Y}\right)$ is called an LF-grill map if, for each $f \in L^{Y}, \mathcal{G}_{X}\left(\phi^{\leftarrow}(f)\right) \leq \mathcal{G}_{Y}(f)$.

Definition 2.3 $[11,17]$ A mapping $\mathcal{C}: L^{X} \rightarrow L^{X}$ is called an $L$-fuzzy closure operator on $X$ if $\mathcal{C}$ satisfies the following conditions: for all $f, g \in L^{X}$

$$
\begin{aligned}
& \mathrm{C} 1 \mathcal{C}\left(\perp_{X}\right)=\perp_{X}, \\
& \mathrm{C} 2 \mathcal{C}(f) \geq f, \\
& \mathrm{C} 3 \text { if } f \leq g, \text { then } \mathcal{C}(f) \leq \mathcal{C}(g), \\
& \mathrm{C} 4 \mathcal{C}(f \vee g) \leq \mathcal{C}(f) \vee \mathcal{C}(g) .
\end{aligned}
$$

The pair $(X, \mathcal{C})$ is called an $L$-fuzzy closure space.

A $L$-fuzzy closure space $(X, \mathcal{C})$ is called:

(1) Topological if $\mathcal{C}(\mathcal{C}(f))=\mathcal{C}(f)$,

(2) stratified if $\mathcal{C}(\alpha \wedge f) \geq \alpha \wedge \mathcal{C}(f)$, for all $\alpha \in L$,

(3) separated if $\mathcal{C}\left(\top_{x}^{*}\right)=\top_{x}^{*}$ for each $x \in X$,

(4) Alexandrov if $\mathcal{C}\left(\bigvee_{i \in \Gamma} f_{i}\right)=\bigvee_{i \in \Gamma} \mathcal{C}\left(f_{i}\right)$ for each subfamily $\left\{f_{i}: i \in \Gamma\right\} \subseteq L^{X}$, 
(5) symmetric if $\mathcal{C}\left(\top_{x}\right)(y)=\mathcal{C}\left(\top_{y}\right)(x)$ for each $y \in X$,

A mapping $\phi:\left(X, \mathcal{C}_{X}\right) \rightarrow\left(Y, \mathcal{C}_{Y}\right)$ between two $L$-fuzzy closure spaces is called $L F$-closure map if $\phi^{\leftarrow}\left(\mathcal{C}_{Y}(h)\right) \geq \mathcal{C}_{X}\left(\phi^{\leftarrow}(h)\right)$ for each $h \in L^{Y}$.

Definition 2.4 [11] A map $\mathcal{F}: L^{X} \rightarrow L$ is called an $L$-fuzzy filter on $X$ if $\mathcal{F}$ satisfies the following conditions for all $f, g \in L^{X}$ :

$$
\begin{array}{ll}
\text { LF1 } & \mathcal{F}\left(\perp_{X}\right)=\perp, \mathcal{F}\left(\top_{X}\right)=\top, \\
\text { LF2 } & \text { if } f \leq g, \text { then } \mathcal{F}(f) \leq \mathcal{F}(g), \\
\text { LF3 } & \mathcal{F}(f \wedge g) \geq \mathcal{F}(f) \wedge \mathcal{F}(g) .
\end{array}
$$

The pair $(X, \mathcal{F})$ is called an $L$-fuzzy filter space. An $L$-fuzzy filter space is called:

(1) Stratified if $\mathcal{F}(\alpha \wedge f) \geq \alpha \wedge \mathcal{F}(f)$ for all $f \in L^{X}$ and $\alpha \in L$.

(2) Separated if $\mathcal{F}\left(T_{x}\right)=T$, for all $x \in X$.

(3) Alexandroff if $\mathcal{F}\left(\bigwedge_{i \in \Gamma} f_{i}\right)=\bigwedge_{i \in \Gamma} \mathcal{F}\left(f_{i}\right)$.

Let $\left(X, \mathcal{F}_{X}\right)$ and $\left(Y, \mathcal{F}_{Y}\right)$ be $L$-fuzzy grill spaces. A mapping $\varphi:\left(X, \mathcal{G}_{X}\right) \rightarrow\left(Y, \mathcal{G}_{Y}\right)$ is called an LF-filter map if, for each $f \in L^{Y}, \mathcal{F}_{X}\left(\phi^{\leftarrow}(f)\right) \geq \mathcal{F}_{Y}(f)$.

Definition 2.5 $[11,16,18]$ A mapping $\mathcal{I}: L^{X} \rightarrow L^{X}$ is called an $L$-fuzzy interior operator on $X$ if $\mathcal{I}$ satisfies the following conditions for all $f, g \in L^{X}$ :

$$
\begin{aligned}
& \text { I1 } \mathcal{I}\left(\top_{X}\right)=\top_{X}, \\
& \text { I2 } \mathcal{I}(f) \leq f, \\
& \text { I3 if } f \leq g \text {, then } \mathcal{I}(f) \leq \mathcal{I}(g), \\
& \text { I4 } \mathcal{I}(f \wedge g) \geq \mathcal{I}(f) \wedge \mathcal{I}(g) .
\end{aligned}
$$

The pair $(X, \mathcal{I})$ is called an $L$-fuzzy interior space.

An $L$-fuzzy interior space $(X, \mathcal{I})$ is called:

(1) Topological if $\mathcal{I}(\mathcal{I}(f))=\mathcal{I}(f)$,

(2) stratified if $\mathcal{I}(\alpha \wedge f) \geq \alpha \wedge \mathcal{I}(f)$,

(3) separated if $\mathcal{I}\left(\top_{x}\right)=\top_{x}$ for each $x \in X$,

(4) Alexandrov if $\mathcal{I}\left(\bigwedge_{i \in \Gamma} f_{i}\right)=\bigwedge_{i \in \Gamma} \mathcal{I}\left(f_{i}\right)$ for each subfamily $\left\{f_{i}: i \in \Gamma\right\} \subseteq L^{X}$.

A mapping $\phi:\left(X, \mathcal{I}_{X}\right) \rightarrow\left(Y, \mathcal{I}_{Y}\right)$ between two $L$-fuzzy interior spaces is called $L I$ map if $\phi^{\leftarrow}\left(\mathcal{I}_{Y}(h)\right) \leq \mathcal{I}_{X}\left(\phi^{\leftarrow}(h)\right)$ for each $h \in L^{Y}$.

Lemma 2.6 Let $\mathcal{F}: L^{X} \rightarrow L$ and $\mathcal{G}: L^{X} \rightarrow L$ be two maps. For all $f \in L^{X}$ and $\alpha \in L$, the following statements are equivalent

(1) $\mathcal{G}(\alpha \wedge f) \geq \alpha \wedge \mathcal{G}(f)($ resp., $\mathcal{F}(\alpha \wedge f) \geq \alpha \wedge \mathcal{F}(f))$

(2) $\mathcal{G}(\alpha \rightarrow f) \leq \alpha \rightarrow \mathcal{G}(f)$ (resp., $\mathcal{F}(\alpha \rightarrow f) \leq \alpha \rightarrow \mathcal{F}(f))$. 
Definition 2.7 [9] A mapping $\delta: L^{X} \times L^{X} \rightarrow L$ is called an $L$-fuzzy pre-proximity on $X$ if it satisfies the following axioms.

P1 $\delta\left(\top_{X}, \perp_{X}\right)=\delta\left(\perp_{X}, \top_{X}\right)=\perp$,

P2 $\delta(f, g) \geq \bigvee_{x \in X}(f \wedge g)(x)$,

P3 If $f_{1} \leq f_{2}, h_{1} \leq h_{2}$, then $\delta\left(f_{1}, h_{1}\right) \leq \delta\left(f_{2}, h_{2}\right)$,

P4 For every $f_{1}, f_{2}, h_{1}, h_{2} \in L^{X}$, we have

$$
\begin{aligned}
& \delta\left(f_{1} \wedge f_{2}, h_{1} \vee h_{2}\right) \leq \delta\left(f_{1}, h_{1}\right) \vee \delta\left(f_{2}, h_{2}\right), \\
& \delta\left(f_{1} \vee f_{2}, h_{1} \wedge h_{2}\right) \leq \delta\left(f_{1}, h_{1}\right) \vee \delta\left(f_{2}, h_{2}\right) .
\end{aligned}
$$

The pair $(X, \delta)$ is called an L-fuzzy pre-proximity space.

An $L$-fuzzy pre-proximity is called stratified if the following hold:

$\delta(\alpha \wedge f, g) \geq \alpha \wedge \delta(f, g)$ and $\delta(f, \alpha \wedge g) \geq \alpha \wedge \delta(f, g)$.

An $L$-fuzzy pre-proximity $\delta$ is called separated if $\delta\left(\top_{x}, \top_{x}^{*}\right)=\delta\left(\top_{x}^{*}, \top_{x}\right)=\perp$ for each $x \in X$.

An $L$-fuzzy pre-proximity is called Alexandroff if

(AL) $\delta\left(\bigvee_{i \in \Gamma} f_{i}, g\right)=\bigvee_{i \in \Gamma} \delta\left(f_{i}, g\right), \quad \delta\left(f, \bigvee_{i \in \Gamma} g_{i}\right)=\bigvee_{i \in \Gamma} \delta\left(f, g_{i}\right)$

Let $\left(X, \delta_{X}\right)$ and $\left(Y, \delta_{Y}\right)$ be two $L$-fuzzy pre-proximity spaces. A mapping $\phi:\left(X, \delta_{X}\right) \rightarrow\left(Y, \delta_{Y}\right)$ is said to be $L$ - pre-proximity map if

$$
\delta_{X}\left(\phi^{\leftarrow}(f), \phi^{\leftarrow}(g)\right) \leq \delta_{Y}(f, g)
$$

Lemma 2.8 An L-fuzzy pre-proximity $\delta$ is stratified if and only if $\delta(\alpha \rightarrow f, g) \leq \alpha \rightarrow \delta(f, g)$ and $\delta(f, \alpha \rightarrow g) \leq \alpha \rightarrow \delta(f, g)$.

Definition $2.9 \quad[19,20]$, A mapping $\mathcal{T}: L^{X} \rightarrow L$ is called an $L$-fuzzy topology on $X$ if it satisfies the following conditions:

LO1 $\mathcal{T}\left(\perp_{X}\right)=\mathcal{T}\left(\top_{X}\right)=\top$,

LO2 $\mathcal{T}\left(f_{1} \wedge f_{2}\right) \geq \mathcal{T}\left(f_{1}\right) \wedge \mathcal{T}\left(f_{2}\right)$, for each $f_{1}, f_{2} \in L^{X}$,

LO3 $\mathcal{T}\left(\bigvee_{i \in \Gamma} f_{i}\right) \geq \bigwedge_{i \in \Gamma} \mathcal{T}\left(f_{i}\right)$, for each $\left\{f_{i}\right\}_{i \in \Gamma} \subseteq L^{X}$.

The pair $(Y, \mathcal{T})$ is called an $L$-fuzzy topological space.

\section{The relationships between $L$-fuzzy pre-proximities and $L$-fuzzy grills}

Now, let $\delta$ be an $L$-fuzzy pre-proximity, we can identify the relation $\delta_{f}$ on $L^{X}$ with the mapping $\delta_{f}: L^{X} \rightarrow L$ such that 


$$
\delta_{f}(g)= \begin{cases}\delta(f, g), & \text { if } g \neq \top_{X} \\ \top, & \text { if } g=\top_{X}\end{cases}
$$

It is clear that $\delta_{f}$ is $L$-fuzzy grill.

Let $\mathcal{P}(X)$ and $\mathcal{G}(X)$ be the families of all $L$-fuzzy pre-proximities and $L$-fuzzy grills on $X$, respectively.

Theorem 3.1 For the mapping $\mathcal{H}: \mathcal{P}(X) \times \mathcal{G}(X) \rightarrow \mathcal{G}(X)$ defined as follows:

$$
\mathcal{H}(\delta, \mathcal{G})(f)=\bigwedge_{g \in L^{X}}(\delta(f, g) \vee \mathcal{G}(f)) .
$$

We have the following properties:

(1) $\mathcal{H}(\delta, \mathcal{G}) \in \mathcal{G}(X)$

(2) $\mathcal{G} \leq \mathcal{H}(\delta, \mathcal{G})$

(3) $\mathcal{H}\left(\delta, \delta_{f}\right)=\delta_{f}$,

(4) If $\delta$ and $\mathcal{G}$ are stratified and Alexandrov, then $\mathcal{H}(\delta, \mathcal{G})$ is stratified and Alexandrov.

Proof (1) (LG1)

$$
\begin{aligned}
& \mathcal{H}(\delta, \mathcal{G})\left(\perp_{X}\right)=\bigwedge_{g \in L^{X}}\left(\delta\left(\perp_{X}, g\right) \vee \mathcal{G}\left(\perp_{X}\right)\right)=\perp, \\
& \mathcal{H}(\delta, \mathcal{G})\left(\top_{X}\right)=\bigwedge_{g \in L^{X}}\left(\delta\left(\top_{X}, g\right) \vee \mathcal{G}\left(\top_{X}\right)\right)=\top .
\end{aligned}
$$

(LG2) Easily proved.

(LG3) Let $f, g \in L^{X}$. Then we have

$$
\begin{aligned}
\mathcal{H}(\delta, \mathcal{G})(f \vee h) & =\bigwedge_{g \in L^{X}}(\delta(f \vee h, g) \vee \mathcal{G}(f \vee h)) \\
& \leq \bigwedge_{g \in L^{X}}((\delta(f, g) \vee \delta(h, g)) \vee(\mathcal{G}(f) \vee \mathcal{G}(h))) \\
& =\bigwedge_{g \in L^{X}}((\delta(f, g) \vee \mathcal{G}(f)) \vee(\delta(h, g) \vee \mathcal{G}(h))) \\
& =\mathcal{H}(\delta, \mathcal{G})(f) \vee \mathcal{H}(\delta, \mathcal{G})(h) .
\end{aligned}
$$

(2) It is clear from the definition.

(3) From (2), $\mathcal{H}\left(\delta, \delta_{f}\right) \geq \delta_{f}$, we need show that $\mathcal{H}\left(\delta, \delta_{f}\right) \leq \delta_{f}$.

$$
\begin{aligned}
\mathcal{H}\left(\delta, \delta_{f}\right)(f) & =\bigwedge_{h \in L^{X}}\left(\delta(h, g) \vee \delta_{f}(g)\right) \\
& \leq \bigwedge_{h \in L^{X}}(\delta(h, g) \vee \delta(f, g)) \\
& \leq \delta(f, g) \vee \delta(f, g) \\
& =\delta(f, g)=\delta_{f} .
\end{aligned}
$$

(4) Let $\alpha \in L$ and $f \in L^{X}$. If $\delta$ and $\mathcal{G}$ are stratified, then we have 


$$
\begin{aligned}
\mathcal{H}(\delta, \mathcal{G})(\alpha \wedge f) & =\bigwedge_{g \in L^{X}}(\delta(\alpha \wedge f, g) \vee \mathcal{G}(\alpha \wedge f)) \\
& \geq \bigwedge_{g \in L^{X}}((\alpha \wedge \delta(f, g)) \vee(\alpha \wedge \mathcal{G}(f))) \\
& =\alpha \wedge \bigwedge_{g \in L^{X}}(\delta(f, g) \vee \mathcal{G}(f)) \\
& =\alpha \wedge \mathcal{H}(\delta, \mathcal{G})(f) .
\end{aligned}
$$

Thus, $\mathcal{H}(\delta, \mathcal{G})$ is stratified.

Let $\left\{f_{i}: i \in \Gamma\right\} \subseteq L^{X}$. If $\delta$ and $\mathcal{G}$ are Alexandrov, then we have

$$
\begin{aligned}
\mathcal{H}(\delta, \mathcal{G})\left(\bigvee_{i \in \Gamma} f_{i}\right) & =\bigwedge_{g \in L^{X}}\left(\delta\left(\bigvee_{i \in \Gamma} f_{i}, g\right) \vee \mathcal{G}\left(\bigvee_{i \in \Gamma} f_{i}\right)\right) \\
& =\bigwedge_{g \in L^{X}}\left(\bigvee_{i \in \Gamma} \delta\left(f_{i}, g\right) \vee \bigvee_{i \in \Gamma} \mathcal{G}\left(f_{i}\right)\right) \\
& =\bigvee_{i \in \Gamma} \bigwedge_{g \in L^{X}}\left(\delta\left(f_{i}, g\right) \vee \mathcal{G}\left(f_{i}\right)\right) \\
& =\bigvee_{i \in \Gamma} \mathcal{H}(\delta, \mathcal{G})\left(f_{i}\right) .
\end{aligned}
$$

Thus, $\mathcal{H}(\delta, \mathcal{G})$ is Alexandrov.

Theorem 3.2 Let $\mathcal{G}$ be L-fuzzy grill on X. Define a map $\mathcal{C}_{\mathcal{G}}: L^{X} \rightarrow L^{X}$ by

$$
\mathcal{C}_{\mathcal{G}}(f)(x)=f(x) \vee \mathcal{G}(f), \quad \forall f \in L^{X}, x \in X .
$$

Then we have the following properties.

(1) $\left(X, \mathcal{C}_{\mathcal{G}}\right)$ is an $L$-fuzzy closure space

(2) If $\mathcal{G}$ is stratified, then $\mathcal{C}_{\mathcal{G}}$ is stratified.

(3) If $\mathcal{G}$ is separated (resp., Alexandrov), then so is $\mathcal{C}_{\mathcal{G}}$.

Theorem 3.3 Let $\mathcal{C}$ be L-fuzzy closure operator on X. Define a map $\mathcal{G}_{\mathcal{C}}: L^{X} \rightarrow L$ by

$$
\mathcal{G}_{\mathcal{C}}(f)=\bigvee_{x \in X} \mathcal{C}(f)(x), \quad \forall f \in L^{X}, x \in X
$$

Then we have the following properties.

(1) $\left(X, \mathcal{G}_{\mathcal{C}}\right)$ is an $L$-fuzzy grill space with $\mathcal{G}_{\mathcal{C}}(f) \geq f(x)$,

(2) If $\mathcal{C}$ is stratified, then $\mathcal{G}_{\mathcal{C}}$ is stratified.

(3) If $\mathcal{C}$ is separated (resp., Alexandrov), then so is $\mathcal{G}_{\mathcal{C}}$,

(4) $\mathcal{G}_{\mathcal{C}_{\mathcal{G}}} \geq \mathcal{G}$ and $\mathcal{C}_{\mathcal{G}_{\mathcal{C}}} \geq \mathcal{C}$.

From the following theorem, we obtain an $L$-fuzzy pre-proximity induced by an $L$-fuzzy grill.

Theorem 3.4 Let $(X, \mathcal{G})$ be an L-fuzzy grill space. Define a map $\delta_{\mathcal{G}}: L^{X} \times L^{X} \rightarrow L$ by 


$$
\delta_{\mathcal{G}}(f, g)=\bigvee_{x \in X}(f(x) \wedge \mathcal{G}(g)), \quad \forall f, g \in L^{X}
$$

such that $\mathcal{G}(g) \geq g(x)$, for all $x \in X$. Then we have the following properties.

(1) $\delta_{\mathcal{G}}$ is an L-fuzzy pre-proximity.

(2) If $\mathcal{G}$ is a stratified, then so is $\delta_{\mathcal{G}}$.

(3) If $\mathcal{G}$ is separated, then $\delta_{\mathcal{G}}$ is separated.

(4) If $\mathcal{G}$ is Alexandroff, then $\delta_{\mathcal{G}}$ is Alexandroff.

Proof (1) (P1) Since $\mathcal{G}\left(\perp_{X}\right)=\perp_{X}$ and $\mathcal{G}\left(\top_{X}\right)=\top_{X}$, we have

$$
\begin{aligned}
& \delta_{\mathcal{G}}\left(\top_{X}, \perp_{X}\right)=\bigvee_{x \in X}\left(\top_{X}(x) \wedge \mathcal{G}\left(\perp_{X}\right)\right)=\perp \\
& \delta_{\mathcal{G}}\left(\perp_{X}, \top_{X}\right)=\bigvee_{x \in X}\left(\perp_{X}(x) \wedge \mathcal{G}\left(\top_{X}\right)\right)=\perp
\end{aligned}
$$

(P2) Since $\mathcal{G}(f) \geq f(x), \forall x \in X$, we have

$$
\begin{aligned}
\delta_{\mathcal{G}}(f, g) & =\bigvee_{x \in X}(f(x) \wedge \mathcal{G}(g)) \\
& \geq \bigvee_{x \in X}(f(x) \wedge g(x))
\end{aligned}
$$

(P3) If $f \leq f_{1}$ and $g \leq g_{1}$, then $\mathcal{G}(g) \leq \mathcal{G}\left(g_{1}\right)$. Thus,

$$
\begin{aligned}
\delta_{\mathcal{G}}(f, g) & =\bigvee_{x \in X}(f(x) \wedge \mathcal{G}(g)) \\
& \leq \bigvee_{x \in X}\left(f_{1}(x) \wedge \mathcal{G}\left(g_{1}\right)\right. \\
& =\delta_{\mathcal{G}}\left(f_{1}, g_{1}\right) .
\end{aligned}
$$

(P4) For every $f_{1}, f_{2}, g_{1}, g_{2} \in L^{X}$, we have

$$
\begin{aligned}
\delta_{\mathcal{G}}\left(f_{1}, g_{1}\right) \vee \delta_{\mathcal{G}}\left(f_{2}, g_{2}\right) & =\left(\bigvee_{x \in X}\left(f_{1}(x) \wedge \mathcal{G}\left(g_{1}\right)\right)\right) \vee\left(\bigvee_{x \in X}\left(f_{2}(x) \wedge \mathcal{G}\left(g_{2}\right)\right)\right) \\
& \geq \bigvee_{x \in X}\left(\left(f_{1}(x) \wedge \mathcal{G}\left(g_{1}\right)\right) \vee\left(f_{2}(x) \wedge \mathcal{G}\left(g_{2}\right)\right)\right) \\
& \geq \bigvee_{x \in X}\left(\left(f_{1}(x) \wedge f_{2}(x)\right) \wedge\left(\mathcal{G}\left(g_{1}\right) \vee \mathcal{G}\left(g_{2}\right)\right)\right) \\
& \geq \bigvee_{x \in X}\left(\left(f_{1} \wedge f_{2}\right)(x) \wedge \mathcal{G}\left(g_{1} \vee g_{2}\right)\right) \\
& =\delta_{\mathcal{G}}\left(f_{1} \wedge f_{2}, g_{1} \vee g_{2}\right),
\end{aligned}
$$

and

$$
\begin{aligned}
\delta_{\mathcal{G}}\left(f_{1} \vee f_{2}, g_{1} \wedge g_{2}\right) & =\bigvee_{x \in X}\left(\left(f_{1} \vee f_{2}\right)(x) \wedge \mathcal{G}\left(g_{1} \wedge g_{2}\right)\right) \\
& \leq \bigvee_{x \in X}\left(\left(f_{1}(x) \vee f_{2}(x)\right) \wedge\left(\mathcal{G}\left(g_{1}\right) \vee \mathcal{G}\left(g_{2}\right)\right)\right) \\
& \leq \bigvee_{x \in X}\left(\left(f_{1}(x) \wedge \mathcal{G}\left(g_{1}\right)\right) \vee\left(f_{2}(x) \wedge \mathcal{G}\left(g_{2}\right)\right)\right) \\
& \leq \bigvee_{x \in X}\left(\left(f_{1}(x) \wedge \mathcal{G}\left(g_{1}\right)\right)\right) \vee \bigvee_{x \in X}\left(\left(f_{2}(x) \wedge \mathcal{G}\left(g_{2}\right)\right)\right) \\
& =\delta_{\mathcal{G}}\left(f_{1}, g_{1}\right) \vee \delta_{\mathcal{G}}\left(f_{2}, g_{2}\right) .
\end{aligned}
$$

Hence, $\delta_{\mathcal{G}}$ is an $L$-fuzzy pre-proximity on $X$. 
(2) If $\mathcal{G}$ is a stratified, we have

$$
\begin{aligned}
\delta_{\mathcal{G}}(f, \alpha \wedge g) & =\bigvee_{x \in X}(f(x) \wedge \mathcal{G}(\alpha \wedge g)) \\
& \geq \bigvee_{x \in X}(f(x) \wedge \alpha \wedge \mathcal{G}(g)) \\
& =\alpha \wedge \bigvee_{x \in X}(f(x) \wedge \mathcal{G}(g)) \\
& =\alpha \wedge \delta_{\mathcal{G}}(f, g),
\end{aligned}
$$

and

$$
\begin{aligned}
\delta_{\mathcal{G}}(\alpha \wedge f, g) & =\bigvee_{x \in X}((\alpha \wedge f)(x) \wedge \mathcal{G}(g)) \\
& =\bigvee_{x \in X}(\alpha \wedge f(x) \wedge \mathcal{G}(g)) \\
& =\alpha \wedge \bigvee_{x \in X}(f(x) \wedge \mathcal{G}(g)) \\
& =\alpha \wedge \delta_{\mathcal{G}}(f, g),
\end{aligned}
$$

for each, $f, g \in L^{X}$ and $\alpha \in L$.

(3) $\delta_{\mathcal{G}}\left(\top_{x}, \top_{x}^{*}\right)=\bigvee_{x \in X} \top_{x}(x) \wedge \mathcal{G}_{\delta}\left(\top_{x}^{*}\right)=\perp$

(4)

$$
\begin{aligned}
\delta_{\mathcal{G}}\left(\bigvee_{i \in \Gamma} f_{i}, g\right) & =\bigvee_{x \in X}\left(\left(\bigvee_{i \in \Gamma} f_{i}\right)(x) \wedge \mathcal{G}(g)\right) \\
& =\bigvee_{x \in X}\left(\bigvee_{i \in \Gamma}\left(f_{i}(x) \wedge \mathcal{G}(g)\right)\right) \\
& =\bigvee_{i \in \Gamma}\left(\bigvee_{x \in X}\left(f_{i}(x) \wedge \mathcal{G}(g)\right)\right) \\
& =\bigvee_{i \in \Gamma} \delta_{\mathcal{G}}\left(f_{i}, g\right)
\end{aligned}
$$

and

$$
\begin{aligned}
\delta_{\mathcal{G}}\left(f, \bigvee_{i \in \Gamma} g_{i}\right) & =\bigvee_{x \in X}\left(f(x) \wedge \mathcal{G}\left(\bigvee_{i \in \Gamma} g_{i}\right)\right) \\
& =\bigvee_{x \in X}\left(f(x) \wedge\left(\bigvee_{i \in \Gamma} \mathcal{G}\left(g_{i}\right)\right)\right) \\
& =\bigvee_{i \in \Gamma}\left(\bigvee_{x \in X}\left(f(x) \wedge \mathcal{G}\left(g_{i}\right)\right)\right) \\
& =\bigvee_{i \in \Gamma} \delta_{\mathcal{G}}\left(f, g_{i}\right) .
\end{aligned}
$$

Thus, $\delta_{\mathcal{G}}$ is Alexandroff.

Corollary 3.5 Let $(X, \mathcal{G})$ be an L-fuzzy grill space. Define a map $\delta_{\mathcal{G}}: L^{X} \times L^{X} \rightarrow L$ by

$$
\delta_{\mathcal{G}}(f, g)=\bigvee_{x \in X}(g(x) \wedge \mathcal{G}(f)), \quad \forall f, g \in L^{X}
$$

such that $\mathcal{G}(f) \geq f(x)$, for all $x \in X$. Then we have the following properties.

(1) $\delta_{\mathcal{G}}$ is an L-fuzzy pre-proximity. 
(2) If $\mathcal{G}$ is a stratified, then so is $\delta_{\mathcal{G}}$.

(3) If $\mathcal{G}$ is separated, then $\delta_{\mathcal{G}}$ is separated.

(4) If $\mathcal{G}$ is Alexandroff, then $\delta_{\mathcal{G}}$ is Alexandroff.

\section{The relationships between $L$-fuzzy pre-proximities and filters}

Now, let $\delta$ be an $L$-fuzzy pre-proximity, we can identify the relation $\mathcal{F}_{f}$ on $L^{X}$ with the mapping $\mathcal{F}_{f}: L^{X} \rightarrow L$ such that

$$
\mathcal{F}_{f}(g)= \begin{cases}\delta^{*}\left(f, g^{*}\right), & \text { if } g \neq \perp_{X} \\ \perp, & \text { if } g=\perp_{X}\end{cases}
$$

It is clear that $\mathcal{F}_{f}$ is $L$-fuzzy filter.

Let $\mathcal{F}(X)$ be the family of all $L$-fuzzy filters on $X$.

Theorem 4.1 For the mapping $\mathcal{H}: \mathcal{P}(X) \times \mathcal{F}(X) \rightarrow \mathcal{F}(X)$ defined as follows:

$$
\mathcal{H}(\delta, \mathcal{F})(f)=\bigvee_{g \in L^{X}}\left(\delta^{*}\left(g, f^{*}\right) \wedge \mathcal{F}(f)\right) .
$$

Then we have the following properties:

(1) $\mathcal{H}(\delta, \mathcal{F}) \in \mathcal{F}(X)$

(2) $\mathcal{H}\left(\delta, \mathcal{F}_{f}\right) \leq \mathcal{F}_{f}$,

(3) $\mathcal{H}\left(\delta, \mathcal{F}_{f}\right)=\mathcal{F}_{f}$,

(4) If $\delta$ and $\mathcal{F}$ are stratified, then $\mathcal{H}(\delta, \mathcal{F})$ is stratified.

Proof (1) (LF1) $\mathcal{H}(\delta, \mathcal{F})\left(\perp_{X}\right)=\bigvee_{g \in L^{X}}\left(\delta^{*}\left(g, \top_{X}\right) \wedge \mathcal{F}\left(\perp_{X}\right)\right)=\perp$,

$\mathcal{H}(\delta, \mathcal{F})\left(\top_{X}\right)=\bigvee_{g \in L^{X}}\left(\delta^{*}\left(g, \perp_{X}\right) \wedge \mathcal{F}\left(\top_{X}\right)\right)=\top$.

(LF2) Easily proved

(LF3) Let $f, g \in L^{X}$. Then we have

$$
\begin{aligned}
\mathcal{H}(\delta, \mathcal{F})(f \wedge h) & =\bigvee_{g \in L^{X}}\left(\delta^{*}\left(g, f^{*} \vee h^{*}\right) \wedge \mathcal{F}(f \wedge h)\right) \\
& \geq \bigvee_{g \in L^{X}}\left(\left(\delta^{*}\left(g, f^{*}\right) \wedge \delta^{*}\left(g, h^{*}\right)\right) \wedge(\mathcal{F}(f) \wedge \mathcal{F}(h))\right) \\
& =\bigvee_{g \in L^{X}}\left(\left(\delta^{*}\left(g, f^{*}\right) \wedge \mathcal{F}(f)\right) \wedge\left(\delta^{*}\left(g, h^{*}\right) \wedge \mathcal{F}(h)\right)\right) \\
& =\mathcal{H}(\delta, \mathcal{F})(f) \wedge \mathcal{H}(\delta, \mathcal{F})(h) .
\end{aligned}
$$

(2) It is clear from the definition

(3) From $(2), \mathcal{H}\left(\delta, \mathcal{F}_{f}\right) \leq \mathcal{F}_{f}$, we need show that $\mathcal{H}\left(\delta, \mathcal{F}_{f}\right) \geq \mathcal{F}_{f}$. 


$$
\begin{aligned}
\mathcal{H}\left(\delta, \mathcal{F}_{f}\right)(g) & =\bigvee_{h \in L^{X}}\left(\delta^{*}\left(h, g^{*}\right) \wedge \mathcal{F}_{f}(g)\right) \\
& =\bigvee_{h \in L^{X}}\left(\delta^{*}\left(h, g^{*}\right) \wedge \delta^{*}\left(f, g^{*}\right)\right) \\
& \geq \delta^{*}\left(f, g^{*}\right) \wedge \delta^{*}\left(f, g^{*}\right) \\
& =\delta^{*}\left(f, g^{*}\right)=\mathcal{F}_{f}(g) .
\end{aligned}
$$

(4) Let $\alpha \in L$ and $f \in L^{X}$. Then we have ,by Lemma 2.8,

$$
\begin{aligned}
\mathcal{H}(\delta, \mathcal{F})(\alpha \wedge f) & =\bigvee_{g \in L^{X}}\left(\delta^{*}\left(g,(\alpha \wedge f)^{*}\right) \wedge \mathcal{F}(\alpha \wedge f)\right) \\
& \geq \bigvee_{g \in L^{X}}\left(\left(\alpha \wedge \delta^{*}\left(g, f^{*}\right)\right) \wedge(\alpha \wedge \mathcal{F}(f))\right) \\
& =\alpha \wedge \bigvee_{g \in L^{X}}\left(\delta^{*}\left(g, f^{*}\right) \wedge \mathcal{F}(f)\right) \\
& =\alpha \wedge \mathcal{H}(\delta, \mathcal{F})(f) .
\end{aligned}
$$

Theorem 4.2 Let $\mathcal{F}$ be an L-fuzzy filter on X. Define a map $\mathcal{I}_{\mathcal{F}}: L^{X} \rightarrow L^{X}$ by

$$
\mathcal{I}_{\mathcal{F}}(f)(x)=f(x) \wedge \mathcal{F}(f), \quad \forall f \in L^{X}, x \in X .
$$

Then we have the following properties.

(1) $\left(X, \mathcal{I}_{\mathcal{F}}\right)$ is an L-fuzzy interior space

(2) If $\mathcal{F}$ is stratified, then $\mathcal{I}_{\mathcal{F}}$ is stratified.

(3) If $\mathcal{F}$ is separated (resp., Alexandrov), then so is $\mathcal{I}_{\mathcal{F}}$.

Theorem 4.3 Let $\mathcal{I}$ be an L-fuzzy interior operator on X. Define a map $\mathcal{F}_{\mathcal{I}}: L^{X} \rightarrow L$ by

$$
\mathcal{F}_{\mathcal{I}}(f)=\bigwedge_{x \in X} \mathcal{I}(f)(x), \quad \forall f \in L^{X}, x \in X .
$$

Then we have the following properties.

(1) $\left(X, \mathcal{F}_{\mathcal{I}}\right)$ is an L-fuzzy filter space with $\mathcal{F}_{\mathcal{I}}(f) \leq f(x)$,

(2) If $\mathcal{I}$ is stratified, then $\mathcal{F}_{\mathcal{I}}$ is stratified.

(3) If $\mathcal{I}$ is separated (resp., Alexandrov), then so is $\mathcal{F}_{\mathcal{I}}$,

(4) $\mathcal{F}_{\mathcal{I}_{\mathcal{F}}} \leq \mathcal{F}$ and $\mathcal{I}_{\mathcal{F}_{\mathcal{I}}} \leq \mathcal{I}$.

Theorem 4.4 Let $\mathcal{F}$ be an $L$-fuzzy filter on X. Define a map $\delta_{\mathcal{F}}: L^{X} \times L^{X} \rightarrow L$ by

$$
\delta_{\mathcal{F}}(f, g)=\bigvee_{x \in X}\left(f(x) \wedge \mathcal{F}^{*}\left(g^{*}\right)\right) \forall f, g \in L^{X} .
$$

such that $\mathcal{F}(f) \leq f(x), \forall x \in X$. Then, we have the following properties:

(1) $\delta_{\mathcal{F}}$ is an L-fuzzy pre-proximity, 
(2) If $\mathcal{F}$ is a stratified then, so is $\delta_{\mathcal{F}}$,

(3) $\mathcal{F} \geq \mathcal{F}_{\delta_{\mathcal{F}}}$,

(4) If $\mathcal{F}$ is separated, then $\delta_{\mathcal{F}}$ is separated,

(5) If $\mathcal{F}$ is Alexandrov, then $\delta_{\mathcal{F}}$ is Alexandrov.

Proof (1) (P1) Since $\mathcal{F}\left(\perp_{X}\right)=\perp_{X}$ and $\mathcal{F}\left(\top_{X}\right)=\top_{X}$, we have

$$
\begin{aligned}
& \delta_{\mathcal{F}}\left(\top_{X}, \perp_{X}\right)=\bigvee_{x \in X}\left(\top_{X}(x) \wedge \mathcal{F}^{*}\left(\perp_{X}^{*}\right)\right)=\perp . \\
& \delta_{\mathcal{F}}\left(\perp_{X}, \top_{X}\right)=\bigvee_{x \in X}\left(\perp_{X}(x) \wedge \mathcal{F}^{*}\left(\top_{X}^{*}\right)\right)=\perp .
\end{aligned}
$$

(P2) Since $\mathcal{F}(g) \leq g(x), \forall x \in X$, we have

$$
\begin{aligned}
\delta_{\mathcal{F}}(f, g) & =\bigvee_{x \in X}\left(f(x) \wedge \mathcal{F}^{*}\left(g^{*}\right)\right) \\
& \geq \bigvee_{x \in X}(f(x) \wedge g(x))
\end{aligned}
$$

(P3) If $g \leq g_{1}, f \leq f_{1}$, then $\mathcal{F}^{*}\left(g^{*}\right) \leq \mathcal{F}^{*}\left(g_{1}^{*}\right)$. Thus,

$$
\begin{aligned}
\delta_{\mathcal{F}}(f, g) & =\bigvee_{x \in X}\left(f(x) \wedge \mathcal{F}^{*}\left(g^{*}\right)\right) \\
& \leq \bigvee_{x \in X}\left(f_{1}(x) \wedge \mathcal{F}^{*}\left(g_{1}^{*}\right)\right) \\
& =\delta_{\mathcal{F}}\left(f_{1}, g_{1}\right) .
\end{aligned}
$$

(T) For $f_{1}, f_{2}, g_{1}, g_{2} \in L^{X}$,

$$
\begin{aligned}
\delta_{\mathcal{F}}\left(f_{1}, g_{1}\right) \vee \delta_{\mathcal{F}}\left(f_{2}, g_{2}\right) & =\bigvee_{x \in X}\left(f_{1}(x) \wedge \mathcal{F}^{*}\left(g_{1}^{*}\right)\right) \vee \bigvee_{x \in X}\left(f_{2}(x) \wedge \mathcal{F}^{*}\left(g_{2}^{*}\right)\right) \\
& \geq \bigvee_{x \in X}\left(f_{1}(x) \wedge f_{2}(x)\right) \wedge\left(\mathcal{F}^{*}\left(g_{1}^{*}\right) \vee \mathcal{F}^{*}\left(g_{2}^{*}\right)\right) \\
& \geq \bigvee_{x \in X}\left(f_{1}(x) \wedge f_{2}(x) \wedge \mathcal{F}^{*}\left(\left(g_{1} \vee g_{2}\right)^{*}\right)\right) \\
& =\delta_{\mathcal{F}}\left(f_{1} \wedge f_{2}, g_{1} \vee g_{2}\right)
\end{aligned}
$$

Hence, $\delta_{\mathcal{F}}$ is an $L$-fuzzy pre-proximity.

(2) If $\mathcal{F}$ is a stratified, by Lemma 2.6, we have $\mathcal{F}^{*}\left(\alpha \rightarrow f^{*}\right) \geq \alpha \wedge \mathcal{F}^{*}\left(f^{*}\right)$.

Thus,

$$
\begin{aligned}
\delta_{\mathcal{F}}(f, \alpha \wedge g) & =\bigvee_{x \in X}\left(f(x) \wedge \mathcal{F}^{*}\left(\alpha \rightarrow g^{*}\right)\right) \\
& \geq \bigvee_{x \in X}\left(f(x) \wedge \alpha \wedge \mathcal{F}^{*}\left(g^{*}\right)\right) \\
& =\alpha \wedge \bigvee_{x \in X}\left(f(x) \wedge \mathcal{F}^{*}\left(g^{*}\right)\right) \\
& =\alpha \wedge \delta_{\mathcal{F}}(f, g) .
\end{aligned}
$$

(3) It is trivial.

(4) Let $\mathcal{F}$ be separated. Then,

$\delta_{\mathcal{F}}\left(\top_{z}, \top_{z}^{*}\right)=\bigvee_{x \in X}\left(\top_{z}(x) \wedge \mathcal{F}^{*}\left(\top_{z}\right)(x)\right)=\perp$. 
(5) It is easily proved from definitions.

Example 4.5 (1) Define $\mathcal{C}_{1}: L^{X} \rightarrow L^{X}$ as $\mathcal{C}_{1}(f)(x)=\bigvee_{x \in X} f(x)$ and $\mathcal{G}_{1}: L^{X} \rightarrow L$ as $\mathcal{G}_{1}(f)=\bigvee_{x \in X} f(x)$. Hence $\mathcal{C}_{1}$ is $L$-fuzzy closure operator on $X$ and $\mathcal{G}_{1}$ is $L$-fuzzy grill on $X$. Since $\mathcal{C}_{1}\left(\top_{x}^{*}\right)=\top_{X}$ and $\mathcal{G}_{1}\left(\top_{x}^{*}\right)=\top_{X}, \mathcal{C}_{1}$ and $\mathcal{G}_{1}$ and are not separated. Theorems 3.2 and 3.3, $\mathcal{C}_{\mathcal{G}_{\mathcal{C}_{1}}} \geq \mathcal{C}_{1}$ and $\mathcal{G}_{\mathcal{C}_{\mathcal{G}_{1}}} \geq \mathcal{G}_{1}$. By Theorem 3.4, we have

$$
\begin{aligned}
\delta_{\mathcal{G}_{1}}(f, g) & =\bigvee_{x \in X}\left(f(x) \wedge \mathcal{G}_{1}(g)\right) \\
& =\bigvee_{x, y \in X}(f(x) \wedge g(y)) .
\end{aligned}
$$

(2) Define $\mathcal{C}_{2}: L^{X} \rightarrow L^{X}$ as $\mathcal{C}_{2}(f)(x)=f(x)$ and $\mathcal{G}_{2}: L^{X} \rightarrow L$ as $\mathcal{G}_{2}(f)=f$, then $\mathcal{C}_{2}$ is $L$-fuzzy closure operator on $X$ and $\mathcal{G}_{2}$ is $L$-fuzzy grill on $X$. Since $\mathcal{C}_{2}\left(\top_{x}^{*}\right)(x)=\top_{x}^{*}$ and $\mathcal{G}_{2}\left(\top_{x}^{*}\right)=\top_{x}^{*}=\perp$, then $\mathcal{C}_{2}$ and $\mathcal{G}_{2}$ are separated. From Theorems 3.2 and 3.3, $\mathcal{C}_{\mathcal{G}_{\mathcal{C}_{2}}} \geq \mathcal{C}_{1}$ and $\mathcal{G}_{\mathcal{G}_{2}} \geq \mathcal{G}_{1}$. By Theorem 3.4, we have

$$
\begin{aligned}
\delta_{\mathcal{G}_{2}}(f, g) & =\bigvee_{x \in X}\left(f(x) \wedge \mathcal{G}_{2}(g)\right) \\
& =\bigvee_{x \in X}(f(x) \wedge g(x))
\end{aligned}
$$

(3) Define $\mathcal{I}_{1}: L^{X} \rightarrow L^{X}$ as $\mathcal{I}_{1}(f)(x)=\bigwedge_{x \in X} f(x)$ and $\mathcal{F}_{1}: L^{X} \rightarrow L$ as $\mathcal{F}_{1}(f)=\bigwedge_{x \in X} f(x)$ Hence $\mathcal{I}_{1}$ is $L$-fuzzy interior operator on $X$ and $\mathcal{F}_{1}$ is $L$-fuzzy filter on $X$. Since $\mathcal{I}_{1}\left(T_{x}\right)=\perp_{X}$ and $\mathcal{F}_{1}\left(T_{x}\right)=\perp, \mathcal{I}_{1}$ and $\mathcal{F}_{1}$ are not separated. By Theorems 4.2 and 4.3 we obtain $\mathcal{I}_{\mathcal{F}_{\mathcal{I}_{1}}} \leq \mathcal{I}_{1}$ and $\mathcal{F}_{\mathcal{I}_{\mathcal{F}_{1}}} \leq \mathcal{F}_{1}$. By Theorem 4.4, we have

$$
\begin{aligned}
\delta_{\mathcal{F}_{2}}(f, g) & =\bigvee_{x \in X}\left(f(x) \wedge \mathcal{F}_{2}^{*}\left(g^{*}\right)\right) \\
& =\bigvee_{x, y \in X}(f(x) \wedge g(y)) .
\end{aligned}
$$

(4) Define $\mathcal{I}_{2}: L^{X} \rightarrow L^{X}$ as $\mathcal{I}_{2}(f)(x)=f(x)$ and $\mathcal{F}_{2}: L^{X} \rightarrow L$ as $\mathcal{I}_{2}(f)=f(x)$. Hence, $\mathcal{I}_{2}$ is $L$-interior operator on $X$ and $\mathcal{F}_{2}$ is $L$ - fuzzy filter. Since $\mathcal{I}_{2}\left(\top_{x}\right)=\top_{x}$ and $\mathcal{F}_{2}\left(\top_{x}\right)=T$, $\mathcal{I}_{2}$ and $\mathcal{F}_{2}$ are separated. By Theorem 4.4, we obtain $L$-fuzzy preproximities $\delta_{\mathcal{I}_{2}}$ as

$$
\begin{aligned}
\delta_{\mathcal{F}_{1}}(f, g) & =\bigvee_{x \in X}\left(f(x) \wedge \mathcal{F}_{1}^{*}\left(g^{*}\right)\right) \\
& =\bigvee_{x \in X}(f(x) \wedge g(x))
\end{aligned}
$$

\section{Galois correspondences}

Theorem 5.1 Let $\left(X, \mathcal{G}_{X}\right)$ and $\left(Y, \mathcal{G}_{Y}\right)$ be L-fuzzy grill spaces and $\phi: X \rightarrow Y$ be a map. If a map $\phi:\left(X, \mathcal{G}_{X}\right) \rightarrow\left(Y, \mathcal{G}_{Y}\right)$ is an LF-grill map, then $\phi:\left(X, \mathcal{C}_{\mathcal{G}_{X}}\right) \rightarrow\left(Y, \mathcal{C}_{\mathcal{G}_{Y}}\right)$ is an LF-closure map.

Proof For each $f \in L^{Y}$, we have 


$$
\begin{aligned}
\mathcal{C}_{\mathcal{G}_{X}}\left(\phi^{\leftarrow}(f)\right)(x) & =\phi^{\leftarrow}(f)(x) \vee \mathcal{G}_{X}\left(\phi^{\leftarrow}(f)\right) \\
& \leq \phi^{\leftarrow}(f)(x) \vee \mathcal{G}_{Y}(f) \\
& =f(\phi(x)) \vee \mathcal{G}_{Y}(f)=\mathcal{C}_{\mathcal{G}_{Y}}(f)(\phi(x)) \\
& =\phi^{\leftarrow}\left(\mathcal{C}_{\mathcal{G}_{Y}}(f)\right)(x) .
\end{aligned}
$$

Theorem 5.2 Let $\left(X, \mathcal{C}_{X}\right)$ and $\left(Y, \mathcal{C}_{Y}\right)$ be L-fuzzy closure spaces and $\phi: X \rightarrow Y$ be a map. If a map $\phi:\left(X, \mathcal{C}_{X}\right) \rightarrow\left(Y, \mathcal{C}_{Y}\right)$ is an LF-closure map, then $\phi:\left(X, \mathcal{G}_{\mathcal{C}_{X}}\right) \rightarrow\left(Y, \mathcal{G}_{\mathcal{C}_{Y}}\right)$ is an LF-grill map.

Proof For each $f \in L^{Y}$, we have

$$
\begin{aligned}
\mathcal{G}_{\mathcal{C}_{X}}\left(\phi^{\leftarrow}(f)\right) & =\bigvee_{x \in X} \mathcal{C}_{X}\left(\phi^{\leftarrow}(f)\right)(x) \\
& \leq \bigvee_{x \in X} \phi^{\leftarrow}\left(\mathcal{C}_{Y}(f)\right)(x) \\
& =\bigvee_{\phi(x) \in Y} \mathcal{C}_{Y}(f)(\phi(x)) \\
& \leq \mathcal{G}_{\mathcal{C}_{Y}}(f) .
\end{aligned}
$$

Theorem 5.3 Let $\left(X, \mathcal{F}_{X}\right)$ and $\left(Y, \mathcal{F}_{Y}\right)$ be L-fuzzy filter spaces and $\phi: X \rightarrow Y$ be a map. If a map $\phi:\left(X, \mathcal{G}_{X}\right) \rightarrow\left(Y, \mathcal{G}_{Y}\right)$ is an LF-filter map, then $\phi:\left(X, \mathcal{I}_{\mathcal{F}_{X}}\right) \rightarrow\left(Y, \mathcal{I}_{\mathcal{F}_{Y}}\right)$ is an LF-interior map.

Proof For each $f \in L^{Y}$, we have

$$
\begin{aligned}
\mathcal{I}_{\mathcal{F}_{X}}\left(\phi^{\leftarrow}(f)\right)(x) & =\phi^{\leftarrow}(f)(x) \wedge \mathcal{F}_{X}\left(\phi^{\leftarrow}(f)\right) \\
& \geq \phi^{\leftarrow}(f)(x) \wedge \mathcal{F}_{Y}(f) \\
& =f(\phi(x)) \wedge \mathcal{F}_{Y}(f)=\mathcal{I}_{\mathcal{F}_{Y}}(f)(\phi(x)) \\
& =\phi^{\leftarrow}\left(\mathcal{I}_{\mathcal{F}_{Y}}(f)\right)(x) .
\end{aligned}
$$

Theorem 5.4 Let $\left(X, \mathcal{I}_{X}\right)$ and $\left(Y, \mathcal{I}_{Y}\right)$ be L-fuzzy interior spaces and $\phi: X \rightarrow Y$ be a map. If a map $\phi:\left(X, \mathcal{I}_{X}\right) \rightarrow\left(Y, \mathcal{I}_{Y}\right)$ is an LF-interior map, then $\phi:\left(X, \mathcal{F}_{\mathcal{I}_{X}}\right) \rightarrow\left(Y, \mathcal{F}_{\mathcal{I}_{Y}}\right)$ is an LF-filter map.

Proof For each $f \in L^{Y}$, we have

$$
\begin{aligned}
\mathcal{F}_{\mathcal{I}_{X}}\left(\phi^{\leftarrow}(f)\right) & =\bigwedge_{x \in X} \mathcal{I}_{X}\left(\phi^{\leftarrow}(f)\right)(x) \\
& \geq \bigwedge_{x \in X} \phi^{\leftarrow}\left(\mathcal{I}_{Y}(f)\right)(x) \\
& =\bigwedge_{x \in X} \mathcal{I}_{Y}(f)(\phi(x)) \\
& =\mathcal{F}_{\mathcal{I}_{Y}}(f) .
\end{aligned}
$$


Theorem 5.5 Let $\left(X, \mathcal{G}_{X}\right)$ and $\left(Y, \mathcal{G}_{Y}\right)$ be L-fuzzy grill spaces and $\phi:\left(X, \mathcal{G}_{X}\right) \rightarrow\left(Y, \mathcal{G}_{Y}\right)$ be an LF-grill map. Then $\phi:\left(X, \delta_{\mathcal{G}_{X}}\right) \rightarrow\left(Y, \delta_{\mathcal{G}_{Y}}\right)$ is an LF-proximity map.

Proof Since $\mathcal{G}_{X}\left(\phi^{\leftarrow}(g)\right) \leq \mathcal{G}_{Y}(g)$, we have

$$
\begin{aligned}
\delta_{\mathcal{G}_{X}}\left(\phi^{\leftarrow}(f), \phi^{\leftarrow}(g)\right) & =\bigvee_{x \in X}\left(\phi^{\leftarrow}(f)(x) \wedge \mathcal{G}_{X}\left(\phi^{\leftarrow}(g)\right)\right) \\
& \left.\leq \bigvee_{x \in X}\left(f(\phi(x)) \wedge \mathcal{G}_{Y}(g)\right)\right) \\
& \left.\leq \bigvee_{y \in Y}\left(f(y) \wedge \mathcal{G}_{Y}(g)\right)\right) \\
& =\delta_{\mathcal{G}_{Y}}(f, g) .
\end{aligned}
$$

Theorem 5.6 Let $\left(X, \mathcal{F}_{X}\right)$ and $\left(Y, \mathcal{F}_{Y}\right)$ be L-fuzzy filter spaces and $\phi:\left(X, \mathcal{F}_{X}\right) \rightarrow\left(Y, \mathcal{F}_{Y}\right)$ be an LF-filter map. Then $\phi:\left(X, \delta_{\mathcal{F}_{X}}\right) \rightarrow\left(Y, \delta_{\mathcal{F}_{Y}}\right)$ is an LF-proximity map.

Proof Since $\mathcal{F}_{Y}(f) \leq \mathcal{F}_{X}\left(\phi^{\leftarrow}(f)\right)$, we have

$$
\begin{aligned}
\delta_{\mathcal{F}_{X}}\left(\phi^{\leftarrow}(f), \phi^{\leftarrow}(g)\right) & =\bigvee_{x \in X}\left(\phi^{\leftarrow}(f)(x) \wedge \mathcal{F}_{X}^{*}\left(\phi^{\leftarrow}\left(g^{*}\right)\right)\right) \\
& \leq \bigvee_{x \in X}\left(f(\phi(x)) \wedge \mathcal{F}_{Y}^{*}\left(g^{*}\right)\right) \\
& \leq \bigvee_{y \in Y}\left(f(y) \wedge \mathcal{F}_{Y}^{*}\left(g^{*}\right)\right) \\
& =\delta_{\mathcal{F}_{Y}}(f, g)
\end{aligned}
$$

Definition 5.7 [21, 22] Suppose that $F: \mathcal{D} \rightarrow \mathcal{C}, G: \mathcal{C} \rightarrow \mathcal{D}$ are concrete functors. The pair $(F, G)$ is called a Galois correspondence between $\mathcal{C}$ and $\mathcal{D}$ if for each $Y \in \mathcal{C}$, $i d_{Y}: F \circ G(Y) \rightarrow Y$ is a $\mathcal{C}$-morphism, and for each $X \in \mathcal{D}, i d_{X}: X \rightarrow G \circ F(X)$ is a $\mathcal{D}$ -morphism.

If $(F, G)$ is a Galois correspondence, then it is easy to check that $F$ is a left adjoint of $G$, or equivalently that $G$ is a right adjoint of $F$.

The category of separated $L$-fuzzy closure spaces with $L F$-closure mappings as morphisms is denoted by SCS.

The category of separated $L$-fuzzy interior spaces with $L F$-interior mappings as morphisms is denoted by SIS.

The category of separated $L$-fuzzy filter spaces (resp. separated $L$-fuzzy grill spaces) with $L$-filter mappings (resp. $L$-grill maps) as morphisms is denoted by SFF (resp. SFG).

From Theorems 3.2 and 5.1, we obtain a concrete functor $\Upsilon:$ SFG $\rightarrow$ SCS defined as

$$
\Upsilon(X, \mathcal{G})=\left(X, \mathcal{C}_{\mathcal{G}}\right), \Upsilon(\phi)=\phi .
$$

From Theorems 3.2 and 5.2, we obtain a concrete functor $\Omega:$ SCS $\rightarrow$ SFG defined as

$$
\Omega(X, \mathcal{C})=\left(X, \mathcal{G}_{\mathcal{C}}\right), \Omega(\phi)=\phi .
$$


Theorem 5.8 $\Omega:$ SFG $\rightarrow$ SFG is a left adjoint of $\Upsilon:$ SGS $\rightarrow$ SFC, i.e., $(\Upsilon, \Omega)$ is a Galois correspondence.

Proof By Theorem 3.3(4), if $\mathcal{G}_{X}$ is an separated $L$-fuzzy grill on a set $X$, then $\Upsilon\left(\Omega\left(\mathcal{G}_{X}\right)\right)=\mathcal{G}_{\mathcal{G}_{\mathcal{G}}} \geq \mathcal{G}_{X}$. Hence, the identity map id $d_{X}:\left(X, \mathcal{G}_{X}\right) \rightarrow\left(X, \mathcal{G}_{\mathcal{C}_{X}}\right)=\left(X, \Upsilon\left(\Omega\left(\mathcal{F}_{X}\right)\right)\right)$ is an $L F$-closure map. Moreover, if $\mathcal{C}_{Y}$ is a separated $L$-fuzzy closure on a set $Y$, by Theorem 3.3(4), $\Omega\left(\Upsilon\left(\mathcal{C}_{Y}\right)\right)=\mathcal{C}_{\mathcal{G}_{\mathcal{C}_{Y}}} \geq \mathcal{C}_{Y}$. Hence the identity map $i d_{Y}:\left(Y, \mathcal{G}_{\mathcal{C}_{\mathcal{G}}}\right) \rightarrow\left(Y, \delta_{Y}\right)$ is $L F$-closure map. Therefore $(\Upsilon, \Omega)$ is a Galois correspondence.

From Theorems 4.2 and 5.3, we obtain a concrete functor $\Theta:$ SFS $\rightarrow$ SFI defined as

$$
\Theta(X, \mathcal{F})=\left(X, \mathcal{I}_{\mathcal{F}}\right), \Theta(\phi)=\phi .
$$

From Theorems 4.3 and 5.4, we obtain a concrete functor $\Gamma: \mathbf{S F I} \rightarrow \mathbf{S F F}$ defined as

$$
\Gamma(X, \mathcal{I})=\left(X, \mathcal{I}_{\mathcal{F}}\right), \Gamma(\phi)=\phi .
$$

Theorem 5.9 $\Gamma:$ SFF $\rightarrow$ SFI is a left adjoint of $\Theta:$ SFI $\rightarrow$ SFF, i.e., $(\Theta, \Gamma)$ is a Galois correspondence.

Proof By Theorem 4.3(4), if $\mathcal{F}_{X}$ is a separated $L$-fuzzy filter on a set $X$, then $\Theta\left(\Gamma\left(\mathcal{F}_{X}\right)\right)=\mathcal{G}_{\mathcal{I}_{\mathcal{F}_{X}}} \leq \mathcal{F}_{X}$. Hence, the identity map $i d_{X}:\left(X, \mathcal{F}_{X}\right) \rightarrow\left(X, \mathcal{G}_{\mathcal{I}_{\mathcal{F}_{X}}}\right)=\left(X, \Theta\left(\Gamma\left(\mathcal{F}_{X}\right)\right)\right)$ is an $L F$-filter map. Moreover, if $\delta_{Y}$ is a separated $L$-fuzzy preproximity on a set $Y$, by Theorem 4.3(4), $\Gamma\left(\Theta\left(\mathcal{I}_{Y}\right)\right)=\mathcal{I}_{\mathcal{F}_{\mathcal{I}_{Y}}} \leq \mathcal{I}_{Y}$. Hence the identity map $i d_{Y}:\left(Y, \Gamma\left(\Theta\left(\mathcal{I}_{Y}\right)\right)\right) \rightarrow\left(Y, \mathcal{I}_{Y}\right)$ is an $L F$-interior map. Therefore $(\Theta, \Gamma)$ is a Galois correspondence. $\square$

\section{L-fuzzy grill fuzzy topological space}

In this section, we assume that $L$ is an order dense chain. Let $\mathcal{T}\left(x_{t}, r\right)=\left\{g \in L^{X}: x_{t} \in g, \mathcal{T}(g) \geq r\right\}$.

Definition 6.1 Let $(X, \mathcal{T})$ be an $L$-fuzzy topological space and $\mathcal{G}$ be an $L$-fuzzy grill on $X$. Then, the triplet $(X, \mathcal{T}, \mathcal{G})$ is called an $L$-fuzzy grill fuzzy topological space.

Definition 6.2 Let $(X, \mathcal{T}, \mathcal{G})$ be an $L$-fuzzy grill fuzzy topological space. The operator $\Phi_{\mathcal{G}, \mathcal{T}}: L^{X} \times L_{\perp} \rightarrow L^{X}$ which defined by:

$$
\Phi_{\mathcal{G}, \mathcal{T}}(f, r)=\bigvee\left\{x_{t} \in P_{t}(X): \mathcal{G}(f \wedge g) \geq r, \text { for each } g \in \mathcal{T}\left(x_{t}, r\right)\right\}
$$

is called the local function associated with $L$-fuzzy grill $\mathcal{G}$ and $L$-fuzzy topology $\mathcal{T}$, simply we denote it by $\Phi_{\mathcal{G}}(f, r)$.

Theorem 6.3 Let $(X, \mathcal{T})$ be an L-fuzzy topological space. Then the following statements hold. 
(1) If $\mathcal{G}$ is an L-fuzzy grill on $X$, then $\Phi_{\mathcal{G}}$ is an increasing function; in the sense that $f \leq g$ implies $\Phi_{\mathcal{G}}(f, r) \leq \Phi_{\mathcal{G}}(g, r)$.

(2) If $\mathcal{G}_{1}$ and $\mathcal{G}_{2}$ are two L-fuzzy grills on $X$ with $\mathcal{G}_{1} \leq \mathcal{G}_{2}$, then $\Phi_{\mathcal{G}_{1}}(f, r) \leq \Phi_{\mathcal{G}_{2}}(f, r)$, $\forall f \in L^{X}, r \in L_{\perp}$.

(3) For any $L$-fuzzy grill $\mathcal{G}$ on $X$, if $\mathcal{G}(f)=\perp$, then $\Phi_{\mathcal{G}}(f, r)=\perp_{X}, \forall r \in L_{\perp}$.

Proof It is clear.

Theorem 6.4 Let $(X, \mathcal{T}, \mathcal{G})$ be an L-fuzzy grill fuzzy topological space. Then for all $f, g \in L^{X}$, we have:

(1) $\Phi_{\mathcal{G}}(f \vee g, r) \geq \Phi_{\mathcal{G}}(f, r) \vee \Phi_{\mathcal{G}}(g, r), r \in L_{\perp}$

(2) $\Phi_{\mathcal{G}}\left(\Phi_{\mathcal{G}}(f, r), r\right) \leq \Phi_{\mathcal{G}}(f, r)=C_{\mathcal{T}}\left(\Phi_{\mathcal{G}}(f, r), r\right) \leq C_{\mathcal{T}}(f, r), r \in L_{\perp}$.

Proof (1) It is clear.

(2) If $x_{t} \notin C_{\mathcal{T}}(f, r)$, then there exists $g \in \mathcal{T}\left(x_{t}, r\right)$ such that $g \wedge f=\perp_{X}$. Then, $\mathcal{G}(g \wedge f, r)=\mathcal{G}\left(\perp_{X}\right)=\perp$. Thus, $x_{t} \notin \Phi_{\mathcal{G}}(f, r)$. Therefore, $\Phi_{\mathcal{G}}(f, r) \leq C_{\mathcal{T}}(f, r)$.

Now, we will show that $C_{\mathcal{T}}\left(\Phi_{\mathcal{G}}(f, r), r\right) \leq \Phi_{\mathcal{G}}(f, r)$. Suppose that $x_{t} \in C_{\mathcal{T}}\left(\Phi_{\mathcal{G}}(f, r), r\right)$, then for every $g \in \mathcal{T}\left(x_{t}, r\right)$ we have $g \wedge \Phi_{\mathcal{G}}(f, r) \neq \perp_{X}$. Let $y_{s} \in g \wedge \Phi_{\mathcal{G}}(f, r)$. Then, $y_{s} \in g$ and $y_{s} \in \Phi_{\mathcal{G}}(f, r)$. Since $y_{s} \in \Phi_{\mathcal{G}}(f, r)$, then for each $h \in L^{X}$ with $y_{s} \in h$ and $\mathcal{T}(h) \geq r$, we have $\mathcal{G}(f \wedge h) \geq r$. Since $y_{s} \in g$ and $\mathcal{T}(g) \geq r$, we have $\mathcal{G}(f \wedge g) \geq r$. Therefore, $x_{t} \in \Phi_{\mathcal{G}}(f, r)$. Thus, $C_{\mathcal{T}}\left(\Phi_{\mathcal{G}}(f, r), r\right) \leq \Phi_{\mathcal{G}}(f, r)$, which implies that, $C_{\mathcal{T}}\left(\Phi_{\mathcal{G}}(f, r), r\right)=\Phi_{\mathcal{G}}(f, r)$. Hence

$$
\Phi_{\mathcal{G}}\left(\Phi_{\mathcal{G}}(f, r), r\right) \leq C_{\mathcal{T}}\left(\Phi_{\mathcal{G}}(f, r), r\right)=\Phi_{\mathcal{G}}(f, r) \leq C_{\mathcal{T}}(f, r) .
$$

Remark 6.5 The following example show that the equality in Theorem 6.4(i) does not always hold.

Example 6.6 Let $X=\{a, b, c, d\}$ and $L=I$. Define an $L$-fuzzy topology $\mathcal{T}: L^{X} \rightarrow L$ on $X$ by:

$$
\mathcal{T}_{1}(f)= \begin{cases}\top, & \text { if } f=\perp_{X}, \top_{X} \\ \frac{1}{2}, & \text { if } f \in\left\{\chi_{\{a\}}, \chi_{\{a, b\}}\right\} \\ \perp, & \text { otherwise, }\end{cases}
$$

Define an $L$-fuzzy grill $\mathcal{G}: L^{X} \rightarrow L$ on $X$ by: 


$$
\mathcal{G}_{1}(f)= \begin{cases}\top, & \text { if } f=\top_{X} \\ \frac{1}{2}, & \text { if } f \in\left\{\chi_{\{a, b, c\}}, \chi_{\{a, b, d\}}\right\} \\ \frac{1}{3}, & \text { if } f \in \chi_{\{a, b\}} \\ \perp, & \text { otherwise, }\end{cases}
$$

Then $(X, \mathcal{T}, \mathcal{G})$ is an $L$-fuzzy grill fuzzy topological space. If $f=\chi_{\{a\}}, g=\chi_{\{b, c\}}$ and $r=\frac{1}{4}$. Then

$\Phi_{\mathcal{G}}(f, r) \vee \Phi_{\mathcal{G}}(g, r)=\perp_{X}<\Phi_{\mathcal{G}}(f \vee g, r)=\chi_{\{a, b, c\}}$

Theorem 6.7 Let $(X, \mathcal{T}, \mathcal{G})$ be an L-fuzzy grill fuzzy topological space. Define the opera$\operatorname{tor} C_{\mathcal{T}}^{\mathcal{G}}: L^{X} \times L_{\perp} \rightarrow L^{X}$ by:

$$
C_{\mathcal{T}}^{\mathcal{G}}(f, r)=f \vee \Phi_{\mathcal{G}}(f, r) .
$$

Then, $C_{\mathcal{T}}^{\mathcal{G}}$ satisfies the following properties:

(1) $C_{\mathcal{T}}^{\mathcal{G}}\left(\perp_{X}, r\right)=\perp_{X}, C_{\mathcal{T}}^{\mathcal{G}}\left(\top_{X}, r\right)=\top_{X}, \forall r \in L_{\perp}$.

(2) $f \leq C_{\mathcal{T}}^{\mathcal{G}}(f, r), \forall f \in L^{X}, r \in L_{\perp}$.

(3) $C_{\mathcal{T}}^{\mathcal{G}}(f, r) \leq C_{\mathcal{T}}^{\mathcal{G}}(f, s)$ if $r \leq s$.

(4) $C_{\mathcal{T}}^{\mathcal{G}}(f \vee g, r \wedge s) \leq C_{\mathcal{T}}^{\mathcal{G}}(f, r) \vee C_{\mathcal{T}}^{\mathcal{G}}(g, s), r, s \in L_{\perp}$.

(5) $C_{\mathcal{T}}^{\mathcal{G}}\left(C_{\mathcal{T}}^{\mathcal{G}}(f, r), r\right)=C_{\mathcal{T}}^{\mathcal{G}}(f, r), r \in L_{\perp}$.

Proof It is straightforward.

Theorem 6.8 Let $(X, \mathcal{T}, \mathcal{G})$ be an L-fuzzy grill fuzzy topological space. Define the map $\mathcal{T}_{\mathcal{G}}: L^{X} \rightarrow$ Lby:

$$
\mathcal{T}_{\mathcal{G}}(f)=\bigvee\left\{r \in L_{\perp}: C_{\mathcal{T}}^{\mathcal{G}}\left(f^{*}, r\right)=f^{*}\right\} .
$$

Then, $\mathcal{T}_{\mathcal{G}}$ is an L-fuzzy topology on $X$.

Proof (LO1) It is clear.

(LO2) Suppose that there exist $f_{1}, f_{2} \in L^{X}$ such that

$$
\mathcal{T}_{\mathcal{G}}\left(f_{1} \wedge f_{2}\right) \nsupseteq \mathcal{T}_{\mathcal{G}}\left(f_{1}\right) \wedge \mathcal{T}_{\mathcal{G}}\left(f_{2}\right) .
$$

By the definitions of $\mathcal{T}_{\mathcal{G}}\left(f_{1}\right)$ and $\mathcal{T}_{\mathcal{G}}\left(f_{2}\right)$, there exist $r_{1}, r_{2} \in L_{\perp}$ with $C_{\mathcal{T}}^{\mathcal{G}}\left(f_{1}^{*}, r\right)=f_{1}^{*}$ and $C_{\mathcal{T}}^{\mathcal{G}}\left(f_{2}^{*}, r\right)=f_{2}^{*}$ such that $\mathcal{T}_{\mathcal{G}}\left(f_{1} \wedge f_{2}\right) \nsucceq r_{1} \wedge r_{2}$. From Theorem 6.7(4),

$$
\begin{aligned}
C_{\mathcal{T}}^{\mathcal{G}}\left(\left(f_{1} \wedge f_{2}\right)^{*}, r_{1} \wedge r_{2}\right) & =C_{\mathcal{T}}^{\mathcal{G}}\left(f_{1}^{*} \vee f_{2}^{*}, r_{1} \wedge r_{2}\right) \\
& \leq C_{\mathcal{T}}^{\mathcal{G}}\left(f_{1}^{*}, r_{1}\right) \vee C_{\mathcal{T}}^{\mathcal{G}}\left(f_{2}^{*}, r_{2}\right) \\
& \leq f_{1}^{*} \vee f_{2}^{*} \\
& =\left(f_{1} \wedge f_{2}\right)^{*} .
\end{aligned}
$$

By Theorem 6.7(2), $C_{\mathcal{T}}^{\mathcal{G}}\left(\left(f_{1} \wedge f_{2}\right)^{*}, r_{1} \wedge r_{2}\right)=\left(f_{1} \wedge f_{2}\right)^{*}$. Then, $\mathcal{T}_{\mathcal{G}}\left(f_{1} \wedge f_{2}\right) \geq r_{1} \wedge r_{2}$. It is contradiction. Hence, $\mathcal{T}_{\mathcal{G}}\left(f_{1} \wedge f_{2}\right) \geq \mathcal{T}_{\mathcal{G}}\left(f_{1}\right) \wedge \mathcal{T}_{\mathcal{G}}\left(f_{2}\right), \forall f_{1}, f_{2} \in L^{X}$. 
(LO3) Suppose that there exist $\left\{f_{i}: i \in \Gamma\right\} \subseteq L^{X}$ such that:

$$
\mathcal{T}_{\mathcal{G}}\left(\bigvee_{i \in \Gamma} f_{i}\right) \nsucceq \bigwedge_{i \in \Gamma} \mathcal{T}_{\mathcal{G}}\left(f_{i}\right) .
$$

Since $L$ is an order dense chain, there exists $r_{0} \in L_{\perp}$ such that

$$
\mathcal{T}_{\mathcal{G}}\left(\bigvee_{i \in \Gamma} f_{i}\right)<r_{0} \leq \bigwedge_{i \in \Gamma} \mathcal{T}_{\mathcal{G}}\left(f_{i}\right)
$$

Since $\bigwedge_{i \in \Gamma} \mathcal{T}_{\mathcal{G}}\left(f_{i}\right) \geq r_{0}$. Then $\mathcal{T}_{\mathcal{G}}\left(f_{i}\right) \geq r_{0}, \forall i \in \Gamma$. This implies that: $C_{\mathcal{T}}^{\mathcal{G}}\left(f_{i}^{*}, r_{0}\right)=f_{i}^{*}$, $\forall i \in \Gamma$. Let $f=\bigvee_{i \in \Gamma} f_{i}$. Then, $f_{i} \leq f, \forall i \in \Gamma$. Therefore, $C_{\mathcal{T}}^{\mathcal{G}}\left(f^{*}, r_{0}\right) \leq C_{\mathcal{T}}^{\mathcal{G}}\left(f_{i}^{*}, r_{0}\right), \forall i \in \Gamma$. Then

$$
\begin{aligned}
C_{\mathcal{T}}^{\mathcal{G}}\left(f^{*}, r_{0}\right) & \leq \bigwedge_{i \in \Gamma} C_{\mathcal{T}}^{\mathcal{G}}\left(f_{i}^{*}, r_{0}\right) \\
& =\bigwedge_{i \in \Gamma} f_{i}^{*} \\
& =\left(\bigvee_{i \in \Gamma} f_{i}\right)^{*} \\
& =f^{*}
\end{aligned}
$$

Thus, $C_{\mathcal{T}}^{\mathcal{G}}\left(f^{*}, r_{0}\right)=f^{*}$. Then, $\mathcal{T}_{\mathcal{G}}\left(\bigvee_{i \in \Gamma} f_{i}\right)=\mathcal{T}_{\mathcal{G}}(f) \geq r_{0}$, a contradiction. Thus, $\mathcal{T}_{\mathcal{G}}\left(\bigvee_{i \in \Gamma} f_{i}\right) \geq \bigwedge_{i \in \Gamma} \mathcal{T}_{\mathcal{G}}\left(f_{i}\right)$, for each $\left\{f_{i}: i \in \Gamma\right\} \subseteq L^{X}$.

Theorem 6.9 Let $(X, \mathcal{T})$ be an L-fuzzy topological space. Then the following statements hold.

(1) If $\mathcal{G}_{1}$ and $\mathcal{G}_{2}$ are L-fuzzy grills on $X$ with $\mathcal{G}_{1} \leq \mathcal{G}_{2}$, then $\mathcal{T}_{\mathcal{G}_{1}} \leq \mathcal{T}_{\mathcal{G}_{2}}$.

(2) If $\mathcal{G}$ is an $L$-fuzzy grill on $X$ and $f \in L^{X}$ with $\mathcal{G}(f)=\perp$, then there exists $r \in L_{\perp}$ such that $\mathcal{T}_{\mathcal{G}}\left(f^{*}\right) \geq r$.

(3) For any $f \in L^{X}, r \in L_{\perp}$ and for any $L$-fuzzy grill $\mathcal{G}$ on $X, \mathcal{T}_{\mathcal{G}}\left(\left(\Phi_{\mathcal{G}}(f, r)\right)^{*}\right) \geq r$.

(4) If $f \in L^{X}, r \in L_{\perp}$ with $\mathcal{T}_{\mathcal{G}}\left(f^{*}\right) \geq r$, then $\Phi_{\mathcal{G}}(f, r) \leq f$.

Proof (1) Let $r \in L_{\perp}$ such that $\mathcal{T}_{\mathcal{G}_{2}}(f) \geq r$. Then $C_{\mathcal{T}}^{\mathcal{G}_{2}}\left(f^{*}, r\right)=f^{*}$. Thus, $f^{*} \vee \Phi_{\mathcal{G}_{2}}\left(f^{*}, r\right)=f^{*}$. This implies that $\Phi_{\mathcal{G}_{2}}\left(f^{*}, r\right) \leq f^{*}$. By Theorem 6.3(2), we have $\Phi_{\mathcal{G}_{1}}\left(f^{*}, r\right) \leq f^{*}$. This implies that $f^{*} \vee \Phi_{\mathcal{G}_{1}}\left(f^{*}, r\right)=f^{*}$. Thus, $C_{\mathcal{T}}^{\mathcal{G}_{1}}\left(f^{*}, r\right)=f^{*}$, which implies that $\mathcal{T}_{\mathcal{G}_{1}}(f) \geq r$. Thus, $\mathcal{T}_{\mathcal{G}_{2}} \leq \mathcal{T}_{\mathcal{G}_{1}}$.

(2) Let $\mathcal{G}$ be an $L$-fuzzy grill, $r \in L_{\perp}$ and $f \in L^{X}$ with $\mathcal{G}(f)=\perp$. Then by Theorem 6.3(3), $\Phi_{\mathcal{G}}(f, r)=\perp_{X}$. Thus $C_{\mathcal{T}}^{\mathcal{G}}(f, r)=f \vee \Phi_{\mathcal{G}}(f, r)=f$. This implies that $\mathcal{T}_{\mathcal{G}}\left(f^{*}\right) \geq r$.

(3) Let $f \in L^{X}$ and $r \in L_{\perp}$. For any $L$-fuzzy grill $\mathcal{G}$ on $X$, we have

$$
C_{\mathcal{T}}^{\mathcal{G}}\left(\Phi_{\mathcal{G}}(f, r), r\right)=\Phi_{\mathcal{G}}(f, r) \vee \Phi_{\mathcal{G}}\left(\Phi_{\mathcal{G}}(f, r), r\right)=\Phi_{\mathcal{G}}(f, r) . \quad \text { (by Theorem 6.4 }(2) \text { ) }
$$

Thus, $\mathcal{T}_{\mathcal{G}}\left(\left(\Phi_{\mathcal{G}}(f, r)\right)^{*}\right) \geq r$.

(4) Let $f \in L^{X}$ and $\quad r \in L_{\perp} \quad$ with $\quad \mathcal{T}_{\mathcal{G}}\left(f^{*}\right) \geq r$. Suppose that $x_{t} \notin f=C_{\mathcal{T}}^{\mathcal{G}}(f, r)=f \vee \Phi_{\mathcal{G}}(f, r)$, which implies that $x_{t} \notin \Phi_{\mathcal{G}}(f, r)$. Thus, $\Phi_{\mathcal{G}}(f, r) \leq f$. 


\section{Acknowledgements}

The authors would like to thank the Editor and anonymous reviewers for their insightful comments and suggestions.

\section{Authors' contributions}

AAR, MAU and AAAE contributes all paper.

\section{Funding}

There are no sources of funding for the research

Availability of data and materials

It is not applicable in our paper.

\section{Competing interests}

We have no competing interests.

\section{Author details}

${ }^{1}$ Department of Mathematics, Faculty of Science, Beni-Suef University, Beni-Suef, Egypt. ${ }^{2}$ Department of Mathematics, Faculty of Science and Arts at Belqarn, University of Bisha, P.O. Box 60, Sabt Al-Alaya 61985, Kingdom of Saudi Arabia.

${ }^{3}$ High Institute of Computer King Marriott, P.O. Box 3135, Alexandria, Egypt.

Received: 7 August 2019 Accepted: 18 September 2020

Published online: 23 October 2020

\section{References}

1. Ghanim, M.H., Tantawy, O.A., Selim, F.M.: On S-fuzzy quasi-proximity spaces. Fuzzy Sets Syst. 109, 285-290 (2000)

2. Shi, F.G.: The category of pointwise S-proximity spaces. Fuzzy Sets Syst. 152, 349-372 (2005)

3. Katsaras, A.K.: Fuzzy proximity spaces. J. Math. Anal. Appl. 68, 100-110 (1979)

4. Katsaras, A.K., Petalas, C.G.: A unified theory of fuzzy topologies, fuzzy proximities and fuzzy uniformities. Rev. Roum. Math. Pures Appl. 28, 845-896 (1983)

5. Katsaras, A.K.: Fuzzy syntopogenous structures compatible with Lowen fuzzy uniformities and Artico-Moresco fuzzy proximities. Fuzzy Sets Syst. 36, 375-393 (1990)

6. Liu, W.J.: Fuzzy proximity spaces redefined. Fuzzy Sets Syst. 15, 241-248 (1985)

7. Artico, G., Moresco, R.: Fuzzy proximities and totally bounded fuzzy uniformities. J. Math. Anal. Appl. 9, 320-1337 (1984)

8. Yue, Y., Shi, F.G.: Generalized quasi-proximities. Fuzzy Sets Syst. 158, 386-398 (2007)

9. Kim, Y.C., Min, K.C.: L-fuzzy proximities and L-fuzzy topologies. Inf. Sci. 173, 93-113 (2005)

10. Höhle, U., Klement, E.P.: Non-classical Logic and Their Applications to Fuzzy Subsets. Kluwer Academic Publishers, Boston (1995)

11. Höhle, U., Rodabaugh, S.E.: Mathematics of Fuzzy Sets: Logic, Topology, and Measure Theory, The Handbooks of Fuzzy Sets Series 3. Kluwer Academic Publishers, Boston (1999)

12. Thron, W.J.: Proximity structures and grills. Math. Ann. 206, 35-62 (1973)

13. Hájek, P.: Metamathematices of Fuzzy Logic. Kluwer Academic Publishers, Dordrecht (1998)

14. Turunen, E.: Mathematics Behind Fuzzy Logic. A Springer-Verlag Co., Heidelberg (1999)

15. Bělohlávek, R.: Fuzzy Relational Systems. Kluwer Academic Publishers, New York (2002)

16. Rodabaugh, S.E., Klement, E.P.: Topological and Algebraic Structures in Fuzzy Sets. The Handbook of Recent Developments in the Mathematics of Fuzzy Sets. Kluwer Academic Publishers, Boston (2003)

17. Oh, J.M., Kim, Y.C.: L-fuzzy fuzzy closure operators, L-fuzzy topologies and L-fuzzy quasi-uniformities. J. Comput. Anal. Appl. 24(5), 910-927 (2018)

18. Ramadan, A.A.: On L-fuzzy interior operators and L-fuzzy quasi-uniform spaces. J. Intell. Fuzzy Syst. 30, 3717-3752 (2016)

19. Šostak, A.P.: On a fuzzy topological structure. Suppl. Rend. Circ. Matem. Palermo2 Ser II 11, 89-103 (1985)

20. Ramadan, A.A.: Smooth topological spaces. Fuzzy Sets Syst. 48, 371-375 (1992)

21. Adámek, J., Herrlich, H., Strecker, G.E.: Abstract and Concrete Categories. Wiley, New York (1990)

22. Zhang, D.: An enriched category approach to many valued topology. Fuzzy Sets Syst. 158, 349-366 (2007)

\section{Publisher's Note}

Springer Nature remains neutral with regard to jurisdictional claims in published maps and institutional affiliations. 